\title{
Quasi-localized states on noncommutative solitons
}

\author{
S. L. Dubovsky, V. A. Rubakov, S. M. Sibiryakov \\ Institute for Nuclear Research of the Russian Academy of Sciences, \\ 60th October Anniversary Prospect, 7a, 117312 Moscow, Russia
}

\begin{abstract}
We consider noncommutative gauge theories which have zero mass states propagating along both commutative and noncommutative dimensions. Solitons in these theories generically carry $U(m)$ gauge group on their world-volume. From the point of view of string theory, these solitons correspond to "branes within branes". We show that once the worldvolume $U(m)$ gauge theory is in the Higgs phase, light states become quasilocalized, rather than strictly localized on the soliton, i.e. they mix with light bulk modes and have finite widths to escape into the noncommutative dimensions. At small values of $U(m)$ symmetry breaking parameters, these widths are small compared to the corresponding masses. Explicit examples considered are adjoint scalar field in the background of a noncommutative vortex in $U(1)$-Higgs theory, and gauge fields in instanton backgrounds in pure gauge noncommutative theories.
\end{abstract}

\section{Introduction and summary}

Quasi-localization, rather than perfect localization, of states on a brane is a common property of various brane-world models [1, 2, 3, 4, 5, 6]. Particles may not be trapped to a brane forever, but may have finite, albeit small, probability to escape into extra dimensions. This phenomenon may occur even at low energies, provided bulk modes have continuum spectrum starting from zero energy and there is mixing between brane modes and continuum modes (for a review see, e.g., Ref. [7]). Clearly, this possibility is of interest for phenomenology, and also for the study of the properties of branes in a more theoretical context.

Particularly interesting field theory branes are noncommutative solitons (for reviews see Refs. [8, 9, 10]). Noncommutative field theory arises in an appropriate limit of string theory [11, and the properties of many noncommutative solitons match nicely the properties of $D$-branes. Indeed, it has been suggested [12 to use the noncommutative solitons for constructing phenomenologically acceptable brane-world models.

In this paper we discuss quasi-localization of states on solitons in noncommutative gauge theories. The class of models we consider is the one where the gauge (and possibly matter) fields have continuum of bulk modes, weakly coupled at low energiest. From the point of view of string theory, solitons in these models correspond to "branes within branes", see, e.g., Ref. [15 and references therein.

\footnotetext{
${ }^{1}$ We live aside unstable solitons corresponding to $D$-branes in tachyonic vacuum [13, 14].
} 
Generically, a soliton of this type has $U(m)$ gauge symmetry on its worldvolume. If (part of) this gauge symmetry is unbroken, charged matter fields as well as massless gauge fields are strictly localized on the soliton. Our main observation is that the situation changes if $U(m)$ on the soliton world-volume is in the Higgs phase: gauge and/or matter fields become quasi-localized. For fields that have massless bulk modes, escape into extra dimensions occurs even at low energies; at small value of the parameter of $U(m)$ symmetry breaking, the life-times against this escape are large compared to the inverse masses of the quasi-localized modes.

To introduce the mechanism of quasi-localization most explicitly, we consider in Sect.2 a simple example of a $m$-vortex solution in $U(1)$ gauge-Higgs theory in two noncommutative and $p$ commutative spatial dimensions [16, 17, 18, 19]. The bulk modes of both gauge and Higgs fields are massive in this model, so escape of these fields into the noncommutative dimensions does not occur at low energies. We introduce an extra adjoint scalar field which has massless modes in the bulk, and show that its states become quasi-localized on the vortex even at low energies, provided that $U(m)$ gauge theory on the vortex is in the Higgs phase. We calculate the widths of the quasi-localized states at small values of the parameter governing $U(m)$ symmetry breaking, and find that these widths are parametrically smaller than the masses of these states. In this model, as well as in another example studied in this paper, there is a hierarchy of life-times of different quasi-localized modes. In the case of vortex, this hierarchy is related to the rotational symmetry of the background: we will see that higher angular momentum modes live longer on the soliton, because certain mixing terms of these modes are either forbidden by rotational symmetry or suppressed due to the centrifugal barrier.

In Sect.3 we discuss quasi-localization on noncommutative solitons in more general terms. We develop perturbation theory in $U(m)$ symmetry breaking parameters and analyze what kind of mixing between brane modes and bulk modes appears to the first and second orders.

In Sect. 4 we study pure gauge theory in four noncommutative and $p$ commutative spatial dimensions. Instantons in this theory [20, 21, 22, 23, 24] correspond to $D p-D(p+4)$ system. "Zero-size" anti-self-dual $m$-instantons in a theory with anti-self-dual noncommutativity [21, 23] (which are actually non-singular solutions) have unbroken, strictly localized $U(m)$ gauge theory on their worldvolume. We show that once the instanton size is non-vanishing, the gauge theory on the instanton world-volume not only is in the Higgs phase, but also becomes quasi-localized even at low energies. We consider explicitly the case of small instanton size, which corresponds to small gauge boson masses on the soliton, and show that the widths of the quasi-localized gauge bosons against the escape into noncommutative dimensions are again small compared to the masses of these states. Whether or not these widths exhibit the hierarchical pattern depends on the structure of the instanton: the hierarchy is absent in examples consid- 
ered in Sect.4, while in another example sketched in Appendix the widths are hierarchical.

It appears that the quasi-localization of low-energy theory on noncommutative solitons is generic in models having massless modes in the bulk and gauge theories in the Higgs phase on the soliton world-volume. It is tempting to speculate that in string theory motivated brane-world models, massive particles which are neutral under electric charge and color may be unstable against escape into extra dimensions. On the other hand, in the context of noncommutative theories without gravity, particles carrying unbroken charges of the brane-world gauge theory, and also massless gauge bosons of that theory are trapped to the solitonic brane forever (in other words, processes like $e^{-} \rightarrow$ nothing or $\gamma \rightarrow$ nothing are

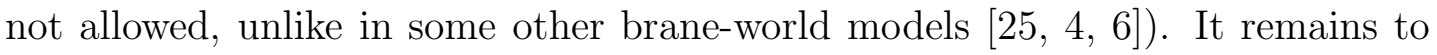
be understood whether or not this property still holds when gravity is turned on.

\section{Quasi-localization on noncommutative vortex}

\section{$2.1 \quad$ Vortex solutions}

In this section we consider an $U(1)$ gauge theory with fundamental Higgs field in $\left(1_{\text {time }}+p+2\right)$-dimensional space-time with two space-like noncommutative dimensions $x_{1}, x_{2}$. The action for this theory has the following form,

$$
S=\frac{1}{g^{2}} \int d^{p+1} y d^{2} x\left[-\frac{1}{4} F_{A B} * F^{A B}+D_{A} \phi^{+} * D^{A} \phi-\frac{1}{2}\left(\phi^{+} * \phi-v^{2}\right)^{2}\right]
$$

where $y^{\mu}$ are commuting dimensions,

$$
\begin{aligned}
F_{A B} & =\partial_{A} A_{B}-\partial_{B} A_{A}-i\left(A_{A} * A_{B}-A_{B} * A_{A}\right) \\
D_{A} \phi & =\partial_{A} \phi-i A_{A} * \phi
\end{aligned}
$$

and the Moyal product is defined as follows,

$$
\left.f(x) * g(x) \equiv \mathrm{e}^{-i \frac{\theta}{2} \epsilon^{i j} \partial_{i} \partial_{j}^{\prime}} f(x) g\left(x^{\prime}\right)\right|_{x=x^{\prime}} .
$$

As shown in Ref. [16, 17, 18, 19], this theory admits static soliton solutions independent of the commuting coordinates and having a form of a vortex in the noncommutative plane $x_{1}, x_{2}$. To describe these solitons, let us switch to the Fock space notations. Then the energy density takes the following form,

$$
E=\frac{2 \pi \theta}{g^{2}} \operatorname{Tr}\left\{\frac{1}{2 \theta^{2}}\left(\left[C, C^{+}\right]+1\right)^{2}+D_{z} \phi^{+} D_{\bar{z}} \phi+D_{\bar{z}} \phi^{+} D_{z} \phi+\frac{1}{2}\left(\phi^{+} \phi-v^{2}\right)^{2}\right\}
$$


where

$$
\begin{gathered}
z=\sqrt{\frac{1}{2}}\left(x_{1}+i x_{2}\right), \quad[z, \bar{z}]=\theta \\
C=a^{+}+i \sqrt{\theta} A_{z} \\
a=\frac{z}{\sqrt{\theta}}, a^{+}=\frac{\bar{z}}{\sqrt{\theta}} \\
D_{z} \phi=-\frac{1}{\sqrt{\theta}}\left[a^{+}, \phi\right]-i A_{z} \phi \\
D_{\bar{z}} \phi=\frac{1}{\sqrt{\theta}}[a, \phi]-i A_{\bar{z}} \phi
\end{gathered}
$$

The properties of stable vortex solutions depend on the value of the parameter $\theta v^{2}$. At $\theta v^{2} \geq 1$ stable vortex is non-BPS and can be obtained by the solution generation technique [18, 19, 23, 26]. The $m$-soliton solution has the following form in this case (for all solitons located at one point in the noncommutative plane)

$$
\begin{aligned}
\phi & =v S_{m}^{+} \\
C^{+} & =S_{m}^{+} a S_{m}
\end{aligned}
$$

where $S_{m}^{+}$is the shift operator,

$$
S_{m}^{+}=\sum_{n=0}^{\infty}|n+m\rangle\langle n| .
$$

In other words, the matrices of the operators $C^{+}$and $\phi$ in the Fock basis have the form

$$
C^{+}=\left(\begin{array}{ccccccc}
0 & 0 & \ldots & 0 & 0 & 0 & \ldots \\
\ldots & \ldots & \ldots & \ldots & \ldots & \ldots \\
0 & 0 & \ldots & 0 & 0 & 0 & \ldots \\
0 & 0 & \ldots & 0 & & & \\
\ldots & \ldots & \ldots & \ldots & & \\
0 & 0 & \ldots & 0 & & &
\end{array}\right) m
$$

$$
\phi=\left(\begin{array}{cccc}
0 & 0 & 0 & \ldots \\
\ldots & \ldots & \ldots \\
0 & 0 & 0 & \ldots \\
v & 0 & 0 & \ldots \\
0 & v & 0 & \ldots \\
0 & 0 & v & \ldots \\
\ldots & \ldots & \ldots
\end{array}\right) m
$$


When considered as a $p$-brane in $(p+2)$-dimensional space, this soliton carries on its world-volume localized gauge fields corresponding to the unbroken $U(m)$ subgroup of the original $U(\infty)$ group. In the Fock basis, the gauge fields of $U(m)$ are $m \times m$ matrices in the upper left corner (cf. Eq. (44)). The corresponding wave functions in the coordinate representation are localized near the vortex and have transverse size of order $\sqrt{\theta}$. There is also continuous spectrum of gauge fields corresponding to the broken generators of $U(\infty)$. The latter fields propagate in the bulk. Non-zero vacuum expectation value of the field $\phi$ at $|z| \rightarrow \infty$ (see Eq. (3)) provides a mass gap $g v$ to this continuum.

At $\theta v^{2}<1$ the solution given by Eq. (3) is unstable. Instead, there exists a BPS solution of the following form [19]

$$
\begin{aligned}
\phi & =v \sum_{n=0}^{\infty}\left(1+\phi_{n}\right)|n+m\rangle\langle n| \\
C^{+} & =\mu_{m}+\epsilon_{m}+S_{m}^{+} \tilde{a} S_{m},
\end{aligned}
$$

where

$$
\begin{gathered}
\mu_{m} \equiv \sum_{\alpha=1}^{m-1} \sqrt{\alpha\left(1-\theta v^{2}\right)}|\alpha-1\rangle\langle\alpha|, \\
\epsilon_{m} \equiv \sqrt{m\left(1-\theta v^{2}\right)}|m-1\rangle\langle m|,
\end{gathered}
$$

and

$$
\tilde{a} \equiv \sum_{n=1}^{\infty}\left(\sqrt{n}+c_{n}\right)|n-1\rangle\langle n| .
$$


The corresponding matrices in the Fock basis are

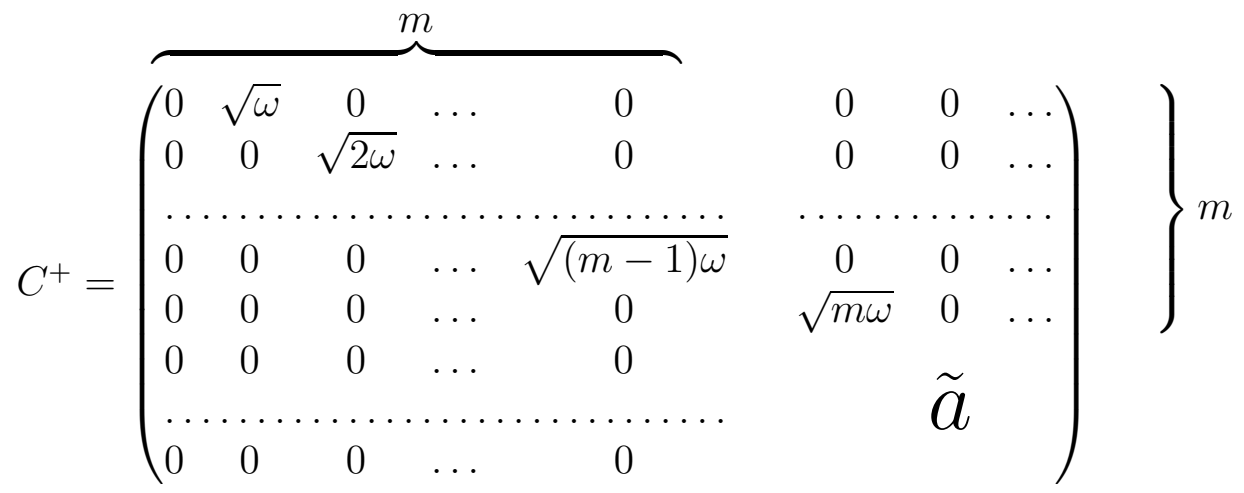

$$
\phi=\left(\begin{array}{cccc}
0 & 0 & 0 & \ldots \\
\ldots & \ldots & \ldots & \ldots \\
0 & 0 & 0 & \ldots \\
v_{1} & 0 & 0 & \ldots \\
0 & v_{2} & 0 & \ldots \\
0 & 0 & v_{3} & \ldots \\
\ldots & \ldots & \ldots
\end{array}\right) \quad{ }^{2} \ldots
$$

where

$$
\omega \equiv \sqrt{1-\theta v^{2}}
$$

and $v_{n}=v\left(1+\phi_{n}\right)$. The coefficients $\phi_{n}$ and $c_{n}$ are determined by a set of recursion relations which were obtained in Ref. [19]. In what follows we consider the case when the parameter $\omega$ is small,

$$
\omega \ll 1 .
$$

In this case $\phi_{n}$ and $c_{n}$ are also small and their explicit form is not essential for our purposes. The relevant property of these coefficients is that they tend to zero as $n$ tends to infinity,

$$
\lim _{n \rightarrow \infty} \phi_{n}, c_{n}=0
$$

$\mu_{m}$ and $\epsilon_{m}$ serve as vacuum expectation values of the adjoint and fundamental Higgs fields giving masses to the gauge bosons of the $U(m)$ gauge group on the vortex. As a result, this gauge group is spontaneously broken completely. In addition, $\epsilon_{m}$ introduces mixing between $U(m)$ gauge bosons and gauge bosons from the continuum spectrum. However, the latter has a mass gap, the gauge bosons from continuum can be integrated out at low energies, and this mixing does not lead to any interesting low energy effects at $\omega \ll 1$. 


\subsection{Adjoint scalar}

Let us now introduce additional massless real adjoint scalar field $f$ with the action

$$
\frac{2 \pi \theta}{g^{2}} \int d^{p+1} y \operatorname{Tr}\left\{-\frac{1}{\theta}[C, f]\left[C^{+}, f\right]+D_{\mu} f D_{\mu} f\right\}
$$

where

$$
D_{\mu} f=\partial_{\mu} f-i\left[A_{\mu}, f\right] .
$$

Let us study the mass spectrum of the field $f$ in the vortex background. A nontrivial mass matrix for $f$ is provided by the first term in the action (13). It is convenient to decompose the field $f$ in the following way,

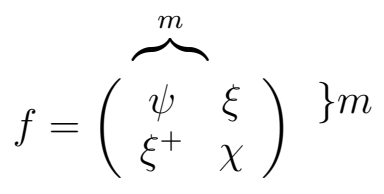

In other words,

$$
f=\psi+\xi+\xi^{+}+\chi
$$

where $\psi$ is a Hermitean $m \times m$ matrix

$$
\psi=P_{m} f P_{m} \equiv \sum_{\alpha, \beta=0}^{m-1} \psi_{\alpha}^{\beta}|\alpha\rangle\langle\beta|,
$$

$\xi$ is a $m \times \infty$ matrix

$$
\xi=P_{m} f\left(1-P_{m}\right) \equiv \sum_{\alpha=0}^{m-1} \sum_{n=0}^{\infty} \xi_{\alpha}^{n}|\alpha\rangle\langle m+n|,
$$

and $\chi$ is a Hermitean $\infty \times \infty$ matrix

$$
\chi=\left(1-P_{m}\right) f\left(1-P_{m}\right) \equiv \sum_{k, n=0}^{\infty} \chi_{k}^{n}|m+k\rangle\langle m+n| .
$$

Here $P_{m}$ is a projector

$$
P_{m}=\sum_{\alpha=0}^{m-1}|\alpha\rangle\langle\alpha|
$$

In the case of non-BPS vortex (3) with unbroken $U(m)$ gauge group on its worldvolume, it is straightforward to check that the fields $\psi_{\alpha}^{\beta}(y)$ are massless scalar fields strictly localized on the vortex world-volume and belonging to adjoint representation of $U(m), \xi_{\alpha}^{n}(y)$ are massive fundamentals with masses

$$
m_{n}^{2}=\frac{2 n+1}{\theta}
$$

and $\chi_{k}^{n}(y)$ are fields in the gapless continuum spectrum, which are neutral under $U(m)$. 


\subsection{Quasi-localization on single vortex}

Let us first discuss the spectrum of the adjoint scalar field at small but non-zero $\omega$ in the background of one-vortex solution, $m=1$. In the matrix notations (10) and (14), the first term in the action (13) takes the following form,

$$
\begin{aligned}
-\frac{1}{\theta} \operatorname{Tr}\left\{[C, f]\left[C^{+}, f\right]\right\}= & -\frac{1}{\theta}\left[\operatorname{Tr}\left\{[\tilde{a}, \chi]\left[\tilde{a}^{+}, \chi\right]\right\}\right. \\
& +\sum_{n=1}^{\infty}\left(2 \tilde{n}_{1}-1\right) \xi^{n-1} \xi_{n-1}^{*}+\sum_{n=1}^{\infty} \omega^{2} \chi_{0}^{n} \chi_{n}^{0}+\omega^{2} \chi_{0}^{0} \chi_{0}^{0}+\omega^{2} \psi^{2} \\
& \left.-\sum_{n=1}^{\infty} 2 \omega \sqrt{\tilde{n}_{2}}\left(\chi_{n}^{0} \xi^{n-1}+\chi_{0}^{n} \xi_{n-1}^{*}\right)-2 \omega^{2} \chi_{0}^{0} \psi\right]
\end{aligned}
$$

where

$$
\tilde{n}_{1,2}=n+O(\omega)
$$

The first term in Eq. (16) describes the propagation of the field $\chi$ along noncommutative dimensions: with appropriate redefinition of noncommutative coordinates it becomes merely the gradient term, $\int d^{2} z^{\prime} \partial_{z^{\prime}} \chi \partial_{\bar{z}^{\prime}} \chi+O(\omega)$. Due to the property (12), the propagation is free far away from the vortex, and the spectrum still starts from zero energy. The second term in Eq. (16) gives large masses (15) (up to small corrections) to the fundamentals $\xi^{n}$. The third, fourth and fifth terms provide small diagonal masses to the fields $\chi_{0}^{n}, \chi_{0}^{0}$ and $\psi$, respectively. Most importantly, there is a term that mixes the would-be localized state $\psi$ with the states from continuum: this is the last term in Eq. (16). Integrating out massive fields $\xi^{n}$ one arrives at the following effective mass terms for light fields $\chi_{n}^{k}$ and $\psi$ (neglecting $O(\omega)$ corrections in $\tilde{n}_{1,2}$ )

$$
\begin{aligned}
M= & -\frac{1}{\theta} \operatorname{Tr}\left\{[\tilde{a}, \chi]\left[\tilde{a}^{+}, \chi\right]\right\}+\sum_{n=0}^{\infty} \frac{\omega^{2}}{\theta}\left(1-\frac{4 n}{2 n-1}\right) \chi_{0}^{n *} \chi_{0}^{n} \\
& -2 \frac{\omega^{2}}{\theta} \chi_{0}^{0} \psi+\frac{\omega^{2}}{\theta} \psi^{2} .
\end{aligned}
$$

This effective mass terms are relevant at energy scales below $1 / \sqrt{\theta}$. One observes that at small $\omega$, there is an interesting low energy scale $\omega / \sqrt{\theta}$, so we concentrate on physics at this scale.

We begin with the first two terms in Eq. (17). They contain corrections to the quadratic action of the fields $\chi_{0}^{n}$, which at first glance appear relevant at the scale $\omega / \sqrt{\theta}$. Let us see that this is not the case. 
Let us come back to coordinate formulation of the noncommutative theory, and write the field $\chi(y, z, \bar{z})$ in Fourier representation along noncommutative dimensions,

$$
\chi(y, z, \bar{z})=\int \frac{d^{2} k}{(2 \pi)^{2}} \tilde{\chi}_{k}(y) e^{i\left(k_{z} z+k_{\bar{z}} \bar{z}\right)} .
$$

We are interested in low-momentum part, $k \sim \omega / \sqrt{\theta}$. The components $\chi_{0}^{n}$ entering the second term in Eq. (17) are

$$
\chi_{0}^{n}=\frac{i^{n}}{\sqrt{n !}} \int \frac{d^{2} k}{(2 \pi)^{2}} \mathrm{e}^{-k^{2} \theta / 2} \tilde{\chi}_{k}(y)\left(k_{\bar{z}} \sqrt{\theta}\right)^{n} \approx \frac{i^{n}}{\sqrt{n !}} \int \frac{d^{2} k}{(2 \pi)^{2}} \tilde{\chi}_{k}(y)\left(k_{\bar{z}} \sqrt{\theta}\right)^{n}
$$

Since $d^{2} k \propto \omega^{2} / \theta$, the second term in Eq. (17) is at least of order $\left(\omega^{6} / \theta^{3} \cdot\left|\tilde{\chi}_{k}\right|^{2}\right)$, whereas the gradient term is of order

$$
\frac{1}{\theta} \int d^{2} k k^{2}\left|\tilde{\chi}_{k}\right|^{2} \sim \omega^{4} / \theta^{3} \cdot\left|\tilde{\chi}_{k}\right|^{2}
$$

Thus, the second term in Eq. (17) is small at small $\omega$ and $k \sim \omega / \sqrt{\theta}$. Similar argument applies to the corrections to the gradient term coming from the fact that $\tilde{a}$ and $a$ differ by $O\left(\omega^{2}\right)$.

Neglecting the higher-order terms, we write the low energy effective action as follows,

$$
\begin{aligned}
S_{e f f}=\frac{2 \pi \theta}{g^{2}} \int d^{p+1} y & \left(\left(\partial_{\mu} \psi\right)^{2}-\frac{\omega^{2}}{\theta} \psi^{2}+\right. \\
& \left.+\int \frac{d^{2} k}{2 \pi \theta(2 \pi)^{2}}\left(\partial_{\mu} \tilde{\chi}_{k} \partial_{\mu} \tilde{\chi}_{-k}-k^{2} \tilde{\chi}_{k} \tilde{\chi}_{-k}+4 \pi \omega^{2} \tilde{\chi}_{k} \psi\right)\right) .
\end{aligned}
$$

From this action one obtains the following equations determining the propagator $G_{\psi}(p)$ of the field $\psi$,

$$
\begin{aligned}
\left(p^{2}-\frac{\omega^{2}}{\theta}\right) G_{\psi}(p)+\frac{\omega^{2}}{\theta} \int \frac{d^{2} k}{(2 \pi)^{2}} \tilde{\chi}_{k} & =1 \\
\left(p^{2}-k^{2}\right) \tilde{\chi}_{k}+2 \pi \omega^{2} G_{\psi}(p) & =0
\end{aligned}
$$

where $p^{\mu}$ is the momentum along commutative dimensions. Expressing $\tilde{\chi}_{k}$ from Eq. (22) and substituting it into Eq. (21) one obtains that the propagator $G_{\psi}(p)$ has the Breit-Wigner form

$$
G_{\psi}(p)=\frac{1}{p^{2}-m^{2}+i m \Gamma}
$$


with mass

$$
m^{2}=\frac{\omega^{2}}{\theta}
$$

and width

$$
\Gamma=-\frac{\omega^{3}}{2 \pi \sqrt{\theta}} \operatorname{Im} \int \frac{d^{2} k}{p^{2}-k^{2}+i \epsilon}=\frac{\pi \omega^{3}}{2 \sqrt{\theta}} \ll m
$$

Thus, mixing between the field $\psi$ and fields $\tilde{\chi}_{k}$ from the continuum spectrum results in the delocalization of the field $\psi$. This field no longer describes a stable state localized on the vortex. Rather it corresponds to a metastable resonance embedded in the continuum spectrum. This state has a small but non-vanishing probability to escape from the brane.

\subsection{Multi-vortex case: hierarchy of widths}

Let us now consider the multi-vortex solution with $m>1$. We still study the case $\omega \ll 1$, and physics at energy scale $\omega / \sqrt{\theta}$. To understand what happens with field $f$ in this case, it is convenient to make use of the symmetry under rotations in the noncommutative plane, which is present in the action (13) when the background field $C^{+}$is given by Eq. (6). Namely, this action is invariant under the transformations

$$
f \rightarrow \mathrm{e}^{-i \alpha a^{+} a} f \mathrm{e}^{i \alpha a^{+} a},
$$

leaving field $C$ invariant. Explicitly, this rotation acts on the matrix elements of the operator $f$ as follows,

$$
f_{m}^{n} \rightarrow \mathrm{e}^{i \alpha(n-m)} f_{m}^{n}
$$

where $f_{m}^{n}$ are defined by

$$
f=\sum_{m, n=0}^{\infty} f_{m}^{n}|m\rangle\langle n| .
$$

In other words, the field $f_{m}^{n}(y)$ has charge $(n-m)$ under this symmetry. Clearly, this charge can be interpreted as the angular momentum in the noncommutative plane. Consequently, the action (13) in the background field (6) is the sum of the actions for fields with different angular momenta. The fields with angular momentum $l$ combine into the matrix

$$
f=\left(\begin{array}{ccccccc}
0 & \ldots & 0 & f_{0}^{l} & 0 & \ldots \ldots \ldots \ldots \\
0 & \ldots & \ldots & 0 & f_{1}^{(l+1)} & 0 & \ldots \ldots \\
0 & \ldots & \ldots & 0 & f_{2}^{(l+2)} & 0 & \ldots \\
\ldots & \ldots & \ldots & \ldots & \ldots
\end{array}\right)+\text { h.c. }
$$


Due to the rotational symmetry, the fields with different $l$ decouple. Furthermore mixing between the states occurs between neighboring entries of this matrix.

In terms of the fields $\psi, \xi$ and $\chi$ introduced in Eq. (14), the latter property implies that the would-be bound states $\psi$ with non-zero angular momentum do not mix directly to the continuum states $\chi$. Indeed, non-trivial mixing occurs between the neighboring entries of the matrix (omitting indices of the fields),

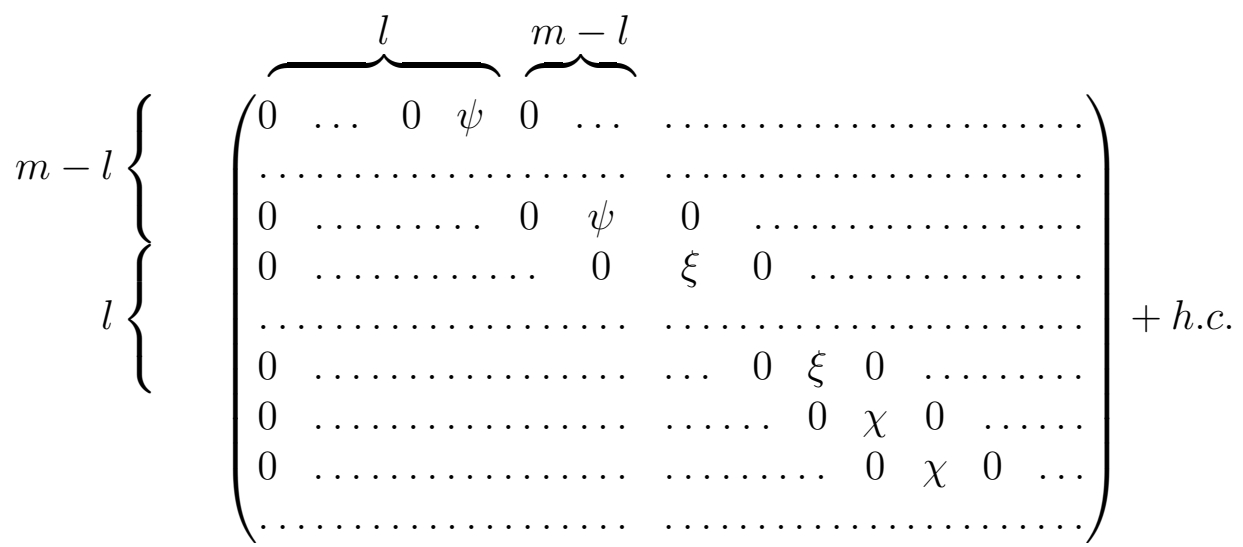

Thus, the fields $\psi$ mix between themselves and with heavy fields $\xi$ only, and the decay of $\psi$ into continuum states occurs only through weak mixing of the heavy fields $\xi$ between themselves and finally with the fields $\chi$ propagating along noncommutative dimensions. Clearly, this introduces the suppression of the decay widths of the quasi-localized states $\psi$ with angular momentum $l$ by extra factor $\omega^{2 l}$. Yet another suppression occurs due to the fact that at low momenta, the components $\chi_{m}^{n}$ with $(n-m)=l \neq 0$ are small, essentially due to the centrifugal barrier.

Explicitly, the mass term for fields with angular momentum $l$ has the following structure,

$$
\begin{aligned}
\theta M_{l}= & -\operatorname{Tr}[a, \chi]\left[a^{+}, \chi\right]-\operatorname{Tr}\left[\epsilon_{m}, \psi\right]\left[\epsilon_{m}^{+}, \psi\right]+\sum_{n=0}^{l-1} m_{n}^{2}\left|\xi_{m+n-l}^{n}\right|^{2} \\
& +\sum_{n=0}^{l-2} \omega \alpha_{n} \xi_{m+n-l}^{n} \xi_{m+n+1-l}^{n+1 *}+\omega^{2} \beta \psi_{m-l-1}^{m-1} \xi_{m-l}^{0 *}+\omega \gamma \xi_{m-1}^{l-1} \chi_{0}^{l *}+\text { h.c. }
\end{aligned}
$$

where $\epsilon_{m}$ is defined in Eq. (8) and is of order $\omega$, the masses $m_{n}$ are the same as in Eq. (15) modulo small corrections proportional to $\omega$, coefficients $\alpha_{n}, \beta$ and $\gamma$ are numbers of order one and we neglected $O\left(\omega^{2}\right)$-corrections involving the components of $\chi$ only. Again, the first term in Eq. (26) provides the kinetic term along the noncommutative plane for the field $\chi$. The second term provides masses and mixings of order $\omega^{2}$ for quasi-localized fields $\psi_{\alpha}^{\beta}$. The third term makes the fields $\xi_{\alpha}^{n}$ heavy. The terms on the second line lead to mixing between quasi-localized modes $\psi$ and heavy fields $\xi$, as well as to mixing between the 
heavy fields and states from continuum. We see, that at $l>0$ there is no direct mixing between $\psi$ and $\chi$. After integrating out the heavy fields, one arrives at the following effective action, describing mixing between the components of $\psi$ and $\chi$ of angular momentum $l$,

$$
\begin{gathered}
S_{e f f}=\sum_{i} \frac{2 \pi \theta}{g^{2}} \int d^{4} y\left(\left|\partial_{\mu} \psi_{i}\right|^{2}-\delta_{i} \frac{\omega^{2}}{\theta}\left|\psi_{i}\right|^{2}\right. \\
\left.+\int \frac{d^{2} k}{2 \pi \theta(2 \pi)^{2}}\left(\partial_{\mu} \tilde{\chi}_{k} \partial_{\mu} \tilde{\chi}_{-k}-k^{2} \tilde{\chi}_{k} \tilde{\chi}_{-k}+\varepsilon_{i} \omega^{l+2}\left(\left(i k_{z} \sqrt{\theta}\right)^{l} \tilde{\chi}_{k}^{*} \psi_{i}+\text { h.c. }\right)\right)\right)
\end{gathered}
$$

here $\psi_{i}$ are eigenvectors of the mass matrix for $\psi$ (the second term in Eq. (26)), $\varepsilon_{i}$ and $\delta_{i}$ are coefficients of order one and the extra factor $\left(k_{z} \sqrt{\theta}\right)^{l}$ is due to the smallness of $\tilde{\chi}_{0}^{l}$ (the centrifugal barrier suppression). Proceeding as before one obtains the following estimate for the widths of the components of the field $\psi$ of angular momentum $l$,

$$
\Gamma \propto \frac{\omega^{4 l+3}}{\sqrt{\theta}} .
$$

We conclude that at small $\theta$, all quasi-localized states have masses of order $\omega / \sqrt{\theta}$, but there is a hierarchy between their widths: modes with higher angular momenta live longer on the soliton.

\section{Perturbative analysis}

Before proceeding to other examples of quasi-localization on noncommutative solitons let us discuss this property in more general terms. The purpose of this treatment is twofold. On the one hand, it helps to elucidate the general character of this phenomenon. On the other hand, it provides a convenient formalism to analyze quasi-localization in more sophisticated setups than the two-dimensional $U(1)$-Higgs model.

Let us consider a gauge theory (possibly with some matter fields) in space of $2 d$ noncommutative coordinates labeled by indices $i, j, k, \ldots$ and $p$ commutative coordinates. Let us assume that this theory admits a soliton which can be obtained by the solution generation technique of [23]. Namely, the gauge field is independent of the commutative coordinates, has vanishing components along commutative coordinates, $A_{\mu}=0, \mu=0,1, \ldots, p$, and has the following form in the Fock basis,

$$
C_{i}^{(0)}=S^{+} a_{i}^{+} S
$$

\footnotetext{
${ }^{2}$ The gauge group of the noncommutative theory need not necessarily be $U(1)$; our analysis applies to noncommutative $U(N)$ gauge theories as well.
} 
Here $a_{i}^{+}, \quad i=1, \ldots, d$, are creation operators and $S$ is a partial isometry operator obeying

$$
\begin{gathered}
S S^{+}=1 \\
S^{+} S=1-P,
\end{gathered}
$$

where $P$ is a projector on the $m$-dimensional subspace $V_{0}$ of the original Fock space. Let $|\alpha\rangle$ be the basis in $V_{0}, \alpha=0,1, \ldots, m-1$. The soliton background (29) leaves unbroken the subgroup $U(m)$ of the gauge group $U(\infty)$; in other words, the soliton carries unbroken $U(m)$ gauge theory on its world-volume. This $U(m)$ may be viewed as the group of unitary transformations in $V_{0}$.

Now, let us further assume that there exists another soliton near (29), whose gauge fields are again independent of the commuting coordinates. If $U(m)$ is in the Higgs phase on the world-volume of that soliton, the gauge fields no longer have the form (29). Instead, they are

$$
C_{i}=S^{+} \tilde{a}_{i}^{+} S+\Delta_{i}^{+}
$$

where $\Delta_{i}^{+}$obeys

$$
S \Delta_{i}^{+} S^{+}=0
$$

In the vortex example of the previous section, $\tilde{a}$ is given by Eq. (9) while $\Delta$ is the sum of (7) and (8).

We assume that a deviation of the gauge field (31) from the field obtained by the solution generation technique is small, so that $\Delta^{+}$and $\left(\tilde{a}_{i}-a_{i}\right)$ may be considered as small perturbations. In addition, we assume that the analogue of the condition (12) is satisfied, i.e.

$$
\left\langle\ldots, n_{i}, \ldots\left|\left(\tilde{a}_{i}-a_{i}\right)\right| \ldots, k_{i}, \ldots\right\rangle \rightarrow 0, \text { as } n_{i}, k_{i} \rightarrow \infty,
$$

where $k_{i}, n_{i}$ are occupation numbers for the $i$-th oscillator.

Let us consider a field $f$ in the adjoint representation of the gauge group of the noncommutative theory. The field $f$ may be a scalar as in the previous section, or it may be a component of the gauge field along the commutative dimensions as will be the case in the next section. For brevity, in what follows we will drop indices corresponding to the Lorentz $S O(1, p)$ group which may be carried by the field $f$. Then the spectrum for the field $f$ is determined by its gradient term along noncommutative coordinates, which has the following form

$$
M_{e f f}=-\frac{1}{\theta} \operatorname{Tr} \sum_{i}\left[C_{i}, f\right]\left[C_{i}^{+}, f\right] .
$$

To study the mass spectrum of the field $f$, let us decompose it in the following way',

$$
f=\psi_{\alpha}^{\beta}|\alpha\rangle\langle\beta|+| \alpha\rangle\left\langle\xi_{\alpha}\left|S+S^{+}\right| \xi_{\alpha}\right\rangle\langle\alpha|+S^{+} \chi S,
$$

\footnotetext{
${ }^{3}$ It is straightforward to check that under conditions (30) every field $f$ can be written in the form (35) in unique way.
} 
where $\left|\xi_{\alpha}\right\rangle$ are arbitrary vectors and $\chi$ is an arbitrary self-conjugate operator. Notations here are similar to Eq. (14).

To the zeroth order in the deviations from the field configuration obtained by the solution generation technique, the gradient term (34) takes the following form,

$$
M_{e f f}^{(0)}=-\frac{1}{\theta} \sum_{i}\left(\operatorname{Tr}\left[a_{i}, \chi\right]\left[a_{i}^{+}, \chi\right]+\left\langle\xi_{\alpha}\left|a_{i}^{+} a_{i}+a_{i} a_{i}^{+}\right| \xi_{\alpha}\right\rangle\right) .
$$

From this equation one finds the usual structure of the mass spectrum for perturbations around the soliton (29): there is a gapless continuum spectrum parametrized by the operator field $\chi$, a discrete spectrum of heavy fields, parametrized by a set of vectors $\left|\xi_{\alpha}\right\rangle$, and a finite number of massless excitations localized on the brane, parametrized by the $m \times m$ matrix $\psi$. From the $p$-dimensional point of view, each vector $\left|\xi_{\alpha}\right\rangle$ corresponds to an infinite tower of fields with masses

$$
m_{n}^{2}=\frac{2 n+1}{\theta}, \quad n=0,1, \ldots
$$

On the other hand, the field $\chi$ has the quadratic action of free $\left(1_{\text {time }}+p+2 d\right)$ dimensional theory, so it freely propagates in both commutative and noncommutative dimensions. To the zeroth order, there is no mixing between $\chi,\left|\xi_{\alpha}\right\rangle$ and $\psi$, so the modes described by $\psi$ and $\left|\xi_{\alpha}\right\rangle$ are strictly localized on the soliton (29). Note also, that if the adjoint field $f$ is interpreted as components $A_{\mu}$ of the gauge field along commutative dimensions, the modes $\psi_{\alpha}^{\beta}$ correspond to the massless $U(m)$ gauge field localized on the soliton.

Let us study perturbation spectrum to higher orders in the deviations from the soliton (29). One feature is that one should replace operators $a_{i}$ in Eq. (33) by operators $\tilde{a}_{i}$. This change introduces mixings between heavy fields $\left|\xi_{\alpha}\right\rangle$ and modifies the gradient term for the field $\chi$ in the vicinity of the soliton. However, this modification will not affect any of the qualitative properties of the spectrum (36) due to the asymptotic condition (33). Furthermore, the arguments similar to those given in the previous section (see Eq. (19)) imply that the corresponding corrections are negligible at small $\left(a_{i}-\tilde{a}_{i}\right)$.

In addition, a number of new terms appear. To the linear order in $\Delta$ these terms are

$$
\begin{aligned}
M_{e f f}^{(1)} & =-\frac{1}{\theta} \sum_{i}\left(2\left\langle\beta\left|\Delta_{i}\right| \alpha\right\rangle\left\langle\xi_{\alpha}\left|\tilde{a}_{i}^{+}\right| \xi_{\beta}\right\rangle\right. \\
& -2\left\langle\xi_{\alpha}\left|\tilde{a}_{i}^{+} \chi S \Delta_{i}\right| \alpha\right\rangle+\left\langle\xi_{\alpha}\left|\chi \tilde{a}_{i}^{+} S \Delta_{i}\right| \beta\right\rangle+\left\langle\alpha\left|\Delta_{i} S^{+} \tilde{a}_{i}^{+} \chi\right| \xi_{\alpha}\right\rangle-2\left\langle\alpha\left|\Delta_{i} S^{+} \chi \tilde{a}_{i}^{+}\right| \xi_{\alpha}\right\rangle \\
& \left.+\psi_{\alpha}^{\beta}\left\langle\beta\left|\Delta_{i} S^{+} \tilde{a}_{i}^{+}\right| \xi_{\alpha}\right\rangle+\psi_{\beta}^{\alpha}\left\langle\xi_{\alpha}\left|\tilde{a}_{i}^{+} S \Delta_{i}\right| \beta\right\rangle+\text { h.c. }\right)
\end{aligned}
$$

This expression is rather lengthy. However, the effects of various terms are quite clear. The first term introduces additional mixing between the heavy fields $\left|\xi_{\alpha}\right\rangle$. 
The four terms in the second line are responsible for mixing between heavy states and continuum spectrum. As a result of this mixing some of the fields $\left|\xi_{\alpha}\right\rangle$ may not be strictly localized on the soliton and rather correspond to quasi-localized heavy fields. The last two terms introduce mixing between the fields $\psi_{\alpha}^{\beta}$ and $\left|\xi_{\alpha}\right\rangle$. The combination of these two effects in general may lead to the non-trivial mixing between the fields $\psi_{\alpha}^{\beta}$ and the states in the continuum spectrum, implying that the localization of the fields $\psi_{\alpha}^{\beta}$ is approximate. In fact, some mixings written in Eq. (37) may vanish in a concrete model. For instance, as we saw in the previous section, there is no mixing, in the vortex background, between the fields $\psi_{\alpha}^{\beta}$ and $\left|\xi_{\alpha}\right\rangle$ to this order.

To the quadratic order in $\Delta$ one has the following mass matrix,

$$
M_{\text {eff }}^{(2)}=-\frac{1}{\theta} \sum_{i} \operatorname{Tr}\left[\Delta_{i}, f\right]\left[\Delta_{i}^{+}, f\right] .
$$

The relevant terms here are as follows. There are mass terms for the fields $\psi_{\alpha}^{\beta}$,

$$
-\frac{1}{\theta} \sum_{i} \operatorname{Tr}\left[\Delta_{i}, \psi\right]\left[\Delta_{i}^{+}, \psi\right] .
$$

Since our analysis applies to the gauge field $A_{\mu}$, these terms give masses to the gauge bosons of the gauge group $U(m)$ residing on the soliton; the (quasi-) localized gauge theory on the soliton is in the Higgs phase. We consider the case of small $\Delta_{i}$, so the masses of these gauge fields are small.

Other terms coming from Eq. (38) are direct mixings between the fields $\psi_{\alpha}^{\beta}$ and the field $\chi$ propagating in the bulk,

$$
-\frac{1}{\theta} \sum_{i} \operatorname{Tr}\left[\Delta_{i}, \psi\right]\left[\Delta_{i}^{+}, S^{+} \chi S\right]+\text { h.c. },
$$

and new mixings between light fields $\psi_{\alpha}^{\beta}$ and heavy fields $\left|\xi_{\alpha}\right\rangle$,

$$
-\frac{1}{\theta} \sum_{i} \operatorname{Tr}\left[\Delta_{i}, \psi\right]\left[\Delta_{i}^{+},\left(S^{+}\left|\xi_{\alpha}\right\rangle\langle\alpha|+| \alpha\rangle\left\langle\xi_{\alpha}\right| S\right)\right]+\text { h.c. . }
$$

In the previous section we saw that the direct mixing between the fields $\psi_{\alpha}^{\beta}$ and continuum spectrum (on the main diagonal, $l=0$ ) and between the heavy fields $\left|\xi_{\alpha}\right\rangle$ and the light fields $\psi_{\alpha}^{\beta}$ appeared only from the second order term (38).

To summarize, around a soliton close to one obtained by the solution generation technique, there is a rich pattern of mixings between would-be localized fields charged under the gauge group on the soliton and the fields propagating in the bulk. These mixings lead to quasi-localization of states on the soliton. Clearly, the necessary condition for these mixings to exist is that there are nonzero matrix elements of the operator $\Delta$ between subspace of zero modes $V_{0}$ and its orthogonal complement. In particular, this condition implies that (part of) the gauge group on the soliton is in the Higgs phase and that only fields which are neutral under the unbroken subgroup may mix with bulk modes. 


\section{Quasi-localization on noncommutative instan- tons}

In this section we study quasi-localization of massive gauge fields on noncommutative instantons. We begin with one-instanton case in $U(2)$ noncommutative gauge theory and make use of the explicit solution found in Ref. [24]. We then generalize to $U(2 k)$ noncommutative gauge theory and consider simple $k$-instantons. These support $U(k)$ gauge theory on their world-volume. Once the $k$-instanton background is such that this $U(k)$ gauge theory is in the Higgs phase, the massive gauge bosons become quasi-localized. For the particular background studied in this section, there is no hierarchy between the widths of these gauge bosons. However, hierarchy may be inherent in some $k$-instanton backgrounds. Indeed, we discuss in Appendix technically more involved example of a two-instanton solution in $U(2)$ noncommutative gauge theory, and show that the widths of the quasi-localized gauge bosons exhibit the hierarchical pattern.

\subsection{One-instanton solution}

Let us describe the one-instanton solution in the $U(2)$ noncommutative pure gauge theory, which was explicitly constructed in Ref. [24]. One considers the $U(2)$ gauge theory in $\left(1_{\text {time }}+p+4\right)$-dimensional space-time with four space-like noncommutative dimensions $z, \bar{z}, \zeta, \bar{\zeta}$ and commutative dimensions $y^{\mu}$. Following Ref. [24] we consider the case of anti-self-dual parameter of noncommutativity, i.e.,

$$
[z, \bar{z}]=-[\zeta, \bar{\zeta}] \equiv \theta>0 .
$$

In the Fock basis, the action for this theory has the following form,

$$
S=(2 \pi \theta)^{2} \int d^{p+1} y \operatorname{Tr}\left[-\frac{1}{4} F_{i j} F^{i j}+\frac{1}{2 \theta} D_{\mu} C_{\bar{\zeta}} D^{\mu} C_{\zeta}+\frac{1}{2 \theta} D_{\mu} C_{\bar{z}} D^{\mu} C_{z}-\frac{1}{4} F_{\mu \nu} F^{\mu \nu}\right]
$$

where $i=z, \bar{\zeta}, C_{i}$ are $2 \times 2$ matrices whose entries are operators acting in the Fock space of two-particle quantum mechanics. Components of the field strength $F_{i j}$ along noncommutative dimensions are determined by these matrices in the usual way

$$
\begin{gathered}
F_{z \bar{z}}=-\frac{1}{\theta}\left(\left[C_{z}, C_{z}^{+}\right]+1\right) \\
F_{\zeta \bar{\zeta}}=\frac{1}{\theta}\left(\left[C_{\bar{\zeta}}, C_{\bar{\zeta}}^{+}\right]+1\right) \\
F_{\zeta z}=-\frac{1}{\theta}\left[C_{\bar{\zeta}}^{+}, C_{z}\right] \\
F_{\zeta \bar{z}}=-\frac{1}{\theta}\left[C_{\bar{\zeta}}^{+}, C_{z}^{+}\right] .
\end{gathered}
$$


The covariant derivative of the field $C_{z}$ is

$$
D_{\mu} C_{z}=\partial_{\mu} C_{z}-i\left[A_{\mu}, C_{z}\right]
$$

and the same for the field $C_{\bar{\zeta}}$. Recall that $C_{\bar{z}}=C_{z}^{+}, C_{\zeta}=C_{\bar{\zeta}}^{+}$, and that the vacuum is $C_{z}=a_{z}^{+} \cdot \mathbf{1}, C_{\bar{\zeta}}=a_{\zeta}^{+} \cdot \mathbf{1}$, where 1 is $2 \times 2$ unit matrix (which we will not write explicitly in what follows).

The instanton solution is independent of commutative coordinates, has $\mu$ components of the gauge field equal to zero and has anti-self-dual field strength. As in the commutative case, the Pontryagin index of the $m$-instanton solution,

$$
N_{P}=-\frac{\theta^{2}}{8} \epsilon^{i j k l} \operatorname{Tr} F_{i j} F_{k l},
$$

is equal to $m$. In Eq. (44) the trace is evaluated over both the $U(2)$ indices and the Fock space.

A powerful tool for describing the moduli space of $m$-instanton solutions and obtaining explicit formulas for instanton fields is the noncommutative version [20] of the ADHM construction [27]. Explicit construction of the one-instanton solution in the noncommutative $U(2)$ theory involves the $4 \times 2$ matrix $\Psi$ [24] which can be written in the following form,

$$
\Psi=\left(\begin{array}{c}
S^{+} \frac{\rho}{\sqrt{N+1+\rho^{2}}} S+1-S^{+} S \\
\frac{\sqrt{N+1}}{\sqrt{N+1+\rho^{2}}} S
\end{array}\right)
$$

where entries are $2 \times 2$ matrices, the matrix $U$ is

$$
S=\frac{1}{\sqrt{N+1}}\left(\begin{array}{cc}
a_{\zeta}^{+} & a_{z} \\
-a_{z}^{+} & a_{\zeta}
\end{array}\right),
$$

and $N$ is the occupation number operator, $N=a_{z}^{+} a_{z}+a_{\zeta}^{+} a_{\zeta}$. The real parameter $\rho$ is natural to interpret as the size of the instanton in units of $\sqrt{\theta}$. The gauge fields $C_{z}(\rho), C_{\bar{\zeta}}(\rho)$ of the instanton of size $\rho$ are

$$
\begin{gathered}
C_{z}(\rho)=\Psi^{+} a_{z}^{+} \Psi \\
C_{\bar{\zeta}}(\rho)=\Psi^{+} a_{\zeta}^{+} \Psi .
\end{gathered}
$$

It is straightforward to check that the field strength obtained from $C_{z}(\rho), C_{\bar{\zeta}}(\rho)$ is anti-self-dual and has unit Pontryagin index [24].

The operator $S$ is a partial isometry operator, i.e.

$$
\begin{gathered}
S S^{+}=1 \\
S^{+} S=1-P_{0},
\end{gathered}
$$


where $P_{0}$ is the projector on the state

$$
|\alpha\rangle=\left(\begin{array}{c}
0 \\
|0\rangle
\end{array}\right) .
$$

The instanton of zero size, $\rho=0$, is non-singular and may be obtained from vacuum by the solution generation technique,

$$
\begin{aligned}
& C_{z}(\rho=0)=S^{+} a_{z}^{+} S \\
& C_{\bar{\zeta}}(\rho=0)=S^{+} a_{\zeta}^{+} S
\end{aligned}
$$

Consequently, the instanton of zero size supports unbroken $U(1)$ gauge group on its world-volume.

\subsection{Quasi-localization on single instanton}

Let us study the spectrum of the components $A_{\mu}$ along the commutative directions, in the background of the instanton of small but non-vanishing size $\rho$. We apply the technique of Sect. 3, with $A_{\mu}$ playing the role of the field $f$.

In the notation of Sect. 3 the operator $\psi$ which describes the would-be zero mode of $A_{\mu}$ is (in what follows we drop the index $\mu$ everywhere)

$$
\psi=\psi_{0}(y)\left(\begin{array}{cc}
0 & 0 \\
0 & |0\rangle\langle 0|
\end{array}\right) .
$$

Now, it is straightforward to present the instanton of non-zero size $\rho$ in the form (31) with

$$
\begin{gathered}
\tilde{a}_{z}=\frac{1}{\sqrt{N+1+\rho^{2}}}\left(\sqrt{N+1} a_{z} \sqrt{N+1}+\rho^{2} S^{+} a_{z} S\right) \frac{1}{\sqrt{N+1+\rho^{2}}} \\
\Delta_{z}=\frac{\rho}{\sqrt{1+\rho^{2}}}|\alpha\rangle\left\langle v_{0}\right|
\end{gathered}
$$

where

$$
\left|v_{0}\right\rangle=\left(\begin{array}{c}
0 \\
a_{z}^{+}|0\rangle
\end{array}\right) .
$$

The expressions for the field $C_{\bar{\zeta}}$ are obtained from Eqs. (52) - (54) by substituting $a_{\zeta}$ for $a_{z}$. Plugging this form of $\Delta$ into the general expression (37) determining the perturbation spectrum about the instanton to the linear order in $\Delta$, one obtains, that the only non-zero term is

$$
M_{\chi \xi}=\frac{2}{\theta} \sum_{i=z, \zeta}\left\langle 0\left|\Delta_{i} S^{+} \chi \tilde{a}_{i}^{+}\right| \xi_{0}\right\rangle .
$$


This term is responsible for the mixing of the heavy field $\left|\xi_{0}\right\rangle$ with the continuum.

This situation is quite similar to what we have found for one-vortex solution in Sect. 2, so let us consider the mass matrix to the second order in $\Delta$, Eq. (38). Among other terms we obtain the mass term for the would-be zero mode $\psi_{0}(y)$ (cf. Eq. (39))

$$
M_{\psi \psi}=\frac{2 \rho^{2}}{\theta\left(1+\rho^{2}\right)} \psi_{0}^{2}
$$

and direct mixing between the field $\psi_{0}(y)$ and continuum (cf. Eq. (40))

$$
M_{\psi \chi}=-\frac{2 \rho^{2}}{\theta\left(1+\rho^{2}\right)} \psi_{0}(\langle\alpha|\chi| \alpha\rangle+\langle u|\chi| u\rangle),
$$

where

$$
|u\rangle=\left(\begin{array}{c}
|0\rangle \\
0
\end{array}\right) .
$$

Since there is no first order mixing between the light mode $\psi$ and heavy modes $\left|\xi_{0}\right\rangle$, mixing involving the heavy states $\left|\xi_{0}\right\rangle$ is negligible at small $\rho$ and low energies.

The rest of the analysis is the same as in the case of vortex. The effect of the two terms (55)), (56) is that the field $\psi_{0}$ describes a quasi-localized massive vector field on the soliton world-volume, whose mass and width (at small $\rho$ ) are

$$
\begin{gathered}
m_{0}^{2}=\frac{2 \rho^{2}}{\theta} \\
\Gamma=\frac{\pi \sqrt{2} \rho^{5}}{\sqrt{\theta}},
\end{gathered}
$$

The extra factor $\rho^{2}$ in the expression for the width, as compared to the case of vortex (cf. Eq. (23), is due to the fact that there are four, rather than two, transverse dimensions in the case of instanton.

\subsection{Multi-instanton case}

In this subsection we discuss quasi-localization on a multi-instanton. Similar to the case of vortex, the main difference from the one-instanton case is that an $m$-instanton solution supports a non-Abelian gauge group $U(m)$ on its worldvolume. If all $m$ instantons sit on top of each other, this $U(m)$ is unbroken. One way to break this gauge group spontaneously is to move to a general point in the Coulomb branch where positions of instantons in the noncommutative hyperplane are not coincident. This splitting leaves unbroken a subgroup $[U(1)]^{m}$ of $U(m)$. The massive gauge bosons on the soliton all carry non-zero charges corresponding to some of the $U(1)$ factors, so they remain strictly localized. 
We consider instead the case in which instantons have non-zero sizes. This corresponds to the Higgs branch of the instanton moduli space. A simple solution of this kind may be obtained in $U(2 k)$ gauge theory by making use of the one-instanton solution considered in the previous subsection. For the sake of simplicity, let us consider two-instanton solution in the $U(4)$ gauge theory; a generalization to $k$-instanton solution in $U(2 k)$ gauge theory is straightforward. The gauge field of a simple anti-self-dual solution describing two instantons of sizes $\rho_{1}$ and $\rho_{2}$ sitting on top of each other has the following block-diagonal form,

$$
C_{z}\left(\rho_{1}, \rho_{2}\right)=\left(\begin{array}{cc}
C_{z}\left(\rho_{1}\right) & 0 \\
0 & C_{z}\left(\rho_{2}\right)
\end{array}\right)
$$

and analogously for $C_{\bar{\zeta}}\left(\rho_{1}, \rho_{2}\right)$. Here $C_{z}\left(\rho_{i}\right)$ are $2 \times 2$ matrices describing oneinstanton solution in the $U(2)$ gauge theory, see Eq. (47). Clearly, the field strength corresponding to Eq. (60) is anti-self-dual and has Pontryagin index equal to two.

When both instantons have zero sizes, $\rho_{1}=\rho_{2}=0$, this solution may be obtained from vacuum by the solution generation technique with the partial isometry operator

$$
S_{2}=\left(\begin{array}{cc}
S & 0 \\
0 & S
\end{array}\right)
$$

where $S$ is given by Eq. (46). In this case there is an unbroken $U(2)$ gauge group, which corresponds to unitary transformations in the two-dimensional subspace $V_{0}$ of the Fock space, whose basis vectors are

$$
\left|\alpha_{1}\right\rangle=\left(\begin{array}{c}
|\alpha\rangle \\
0
\end{array}\right), \quad\left|\alpha_{2}\right\rangle=\left(\begin{array}{c}
0 \\
|\alpha\rangle
\end{array}\right)
$$

where the two-column $|\alpha\rangle$ is given by Eq. (49). The four real zero modes $\psi$ of the field $A_{\mu}$, corresponding to this gauge group, can be organized as follows,

$$
\psi=\left(\begin{array}{ll}
\psi_{1}^{1}(y) & \psi_{1}^{2}(y) \\
\psi_{2}^{1}(y) & \psi_{2}^{2}(y)
\end{array}\right) \otimes|\alpha\rangle\langle\alpha|
$$

where

$$
\psi_{1}^{2}=\psi_{2}^{1 *}
$$

and $\psi_{1}^{1}, \psi_{2}^{2}$ are real.

When both instantons have small but non-zero sizes, $\rho_{1} \neq 0, \rho_{2} \neq 0$, the $U(2)$ gauge group is completely Higgsed, and all its gauge fields become massive. Their mass matrix is obtained by plugging the fields (63) and (60) into Eq. (39) with the result

$$
M_{\psi \psi}=\frac{2}{\theta}\left(\frac{\rho_{1}^{2}}{1+\rho_{1}^{2}}\left(\psi_{1}^{1}\right)^{2}+\frac{\rho_{2}^{2}}{1+\rho_{2}^{2}}\left(\psi_{2}^{2}\right)^{2}+\left(\frac{\rho_{1}^{2}}{1+\rho_{1}^{2}}+\frac{\rho_{2}^{2}}{1+\rho_{2}^{2}}\right) \psi_{1}^{2} \psi_{2}^{1}\right)
$$


Similarly to the one-instanton case, there is no mixing between the fields $\psi_{\alpha}^{\beta}$ and heavy charged fields $\left|\xi_{\alpha}\right\rangle$ to the linear order and the leading contributions to the widths of $\psi_{\alpha}^{\beta}$ come from direct mixing with the fields from the continuum, Eq. (40). The diagonal components $\psi_{1}^{1}$ and $\psi_{2}^{2}$ mix with the corresponding diagonal components $\chi_{1}^{1}$ and $\chi_{2}^{2}$. Each of these mixings has precisely the same form as in the one-instanton case, Eq. (56), leading to the widths

$$
\Gamma_{11}=\frac{\pi \sqrt{2} \rho_{1}^{5}}{\sqrt{\theta}}, \quad \Gamma_{22}=\frac{\pi \sqrt{2} \rho_{2}^{5}}{\sqrt{\theta}} .
$$

The off-diagonal component $\psi_{1}^{2}$ mixes with the off-diagonal component $\chi_{2}^{1}$ of the field $\chi$. This mixing has the following form

$$
M_{\psi \chi}^{o f f-d i a g}=-\frac{2 \rho_{1} \rho_{2}}{\theta \sqrt{\left(1+\rho_{1}^{2}\right)\left(1+\rho_{2}^{2}\right)}} \psi_{1}^{2}\left(\left\langle\alpha\left|\chi_{2}^{1}\right| \alpha\right\rangle+\left\langle u\left|\chi_{2}^{1}\right| u\right\rangle\right)+\text { h.c. },
$$

where $|u\rangle$ is still given by Eq. (57). This mixing leads to the width of $\psi_{1}^{2}$,

$$
\Gamma_{12}=\frac{\pi \sqrt{2}\left(\rho_{1} \rho_{2}\right)^{2}\left(\rho_{1}^{2}+\rho_{2}^{2}\right)^{1 / 2}}{\sqrt{\theta}} .
$$

We see that when one of the instantons has zero size, one of the diagonal gauge bosons is massless, which is related to the fact that the $U(2)$ gauge group on the instanton world-volume is broken down to its $U(1)$ subgroup. In this case the off-diagonal component of the vector field is massive, see Eq. (64). However, its width is equal to zero. This result is in complete agreement with our general reasoning in section 3, as this component describes massive vector field charged under the unbroken $U(1)$ gauge group in this case.

If $\rho_{1}$ and $\rho_{2}$ are of the same order, $\rho_{1} \sim \rho_{2} \sim \rho$, there is no hierarchy between the masses and widths of the quasi-localized gauge bosons: all three masses are of order $\rho / \sqrt{\theta}$, and all three widths are smaller by a factor $\rho^{4}$. The hierarchy of widths may be inherent in other noncommutative instanton backgrounds: in Appendix we present a two-instanton solution in whose background the widths of quasi-localized gauge bosons exhibit the hierarchy similar to one appearing in the multi-vortex case.

\section{Acknowledgments}

The authors are indebted to F.L. Bezrukov for useful discussions. This work has been supported in part by RFBR grant 99-02-18410, CPG and SSLSS grant 00-1596626, CRDF grant (award RP1-2103), Swiss Science Foundation grant 7SUPJ062239. The work of S.S. was supported in part also under RFBR grant 01-02-06034. 


\section{A Quasi-localization in two-instanton background in noncommutative $U(2)$ gauge theory}

To obtain an explicit two-instanton solution in noncommutative $U(2)$ gauge theory, we make use of the ADHM construction. We begin with the following solution of the ADHM equations,

$$
\begin{gathered}
B_{1}=\left(\begin{array}{ll}
0 & 0 \\
\rho & 0
\end{array}\right) \quad B_{2}=\left(\begin{array}{ll}
0 & \rho \\
0 & 0
\end{array}\right) \\
I=\left(\begin{array}{ll}
\rho & 0 \\
0 & \rho
\end{array}\right) \quad J=\left(\begin{array}{cc}
\rho & 0 \\
0 & -\rho
\end{array}\right)
\end{gathered}
$$

With these ADHM data, one should find two "zero modes" $\Psi^{a}(a=1,2)$ which are solutions of the following equation

$$
\mathcal{D} \Psi^{a}=0
$$

where

$$
\mathcal{D}=\left(\begin{array}{c}
\tau \\
\sigma^{+}
\end{array}\right)
$$

is a $4 \times 6$ matrix whose $2 \times 6$ entries are

$$
\tau=\left(B_{2}-a_{z}, \quad B_{1}-a_{\zeta}^{+}, \quad I\right)
$$

and

$$
\sigma^{+}=\left(\begin{array}{lll}
-\left(B_{1}^{+}-a_{\zeta}\right), & B_{2}^{+}-a_{z}^{+}, & J^{+}
\end{array}\right) .
$$

The two linear independent solutions to Eq. (70) are

$$
\Psi^{1}=\left(\begin{array}{c}
0 \\
\sqrt{2} \rho a_{-}^{+} a_{+}^{+} \\
2 \rho^{2} a_{+}^{+} \\
\sqrt{2} \rho\left(2 \rho^{2}+N_{-}+1\right) \\
\sqrt{2} \rho\left(a_{+}^{+}\right)^{2} \\
\left(2 \rho^{2}+N_{+}+N_{-}+1\right) a_{-}^{+}
\end{array}\right) \cdot \mathcal{N}_{1}
$$

and

$$
\Psi^{2}=\left(\begin{array}{c}
\sqrt{2} \rho\left(2 \rho^{2}+N_{-}\right) \\
2 \rho^{2} a_{+} \\
-\sqrt{2} \rho a_{+} a_{-} \\
0 \\
-a_{-}\left(2 \rho^{2}+N_{+}+N_{-}\right) \\
\sqrt{2} \rho a_{+}^{2}
\end{array}\right) \cdot \mathcal{N}_{2}
$$


where

$$
\begin{gathered}
a_{ \pm}=\frac{a_{\zeta} \pm a_{z}}{\sqrt{2}}, \\
N_{ \pm}=a_{ \pm}^{+} a_{ \pm},
\end{gathered}
$$

$\mathcal{N}_{1,2}$ are normalization factors and $\rho$ is a real parameter which may be interpreted as the instanton size. We will need the expression for $\mathcal{N}_{2}$ only,

$$
\begin{aligned}
\mathcal{N}_{2}=\left[2 \rho^{2}\left(2 \rho^{2}+N_{-}\right)^{2}+\left(2 \rho^{2}\right)^{2} N_{+}+2 \rho^{2} N_{+} N_{-}+\right. & \left(N_{+}+N_{-}+2 \rho^{2}\right)^{2} N_{-} \\
& \left.+2 \rho^{2} N_{+}\left(N_{+}-1\right)\right]^{-\frac{1}{2}}
\end{aligned}
$$

Let us first consider the limit $\rho \rightarrow 0$. In this limit the solutions (71) and (72) take the following form,

$$
\Psi_{(0)}^{1}=\left(\begin{array}{c}
0 \\
0 \\
0 \\
0 \\
0 \\
a_{-}^{+} \frac{1}{\sqrt{N_{-}+1}}
\end{array}\right)
$$

and

$$
\Psi_{(0)}^{2}=\left(\begin{array}{c}
\left|0_{+}, 0_{-}\right\rangle\left\langle 0_{+}, 0_{-}\right| \\
\left|0_{+}, 0_{-}\right\rangle\left\langle 1_{+}, 0_{-}\right| \\
0 \\
0 \\
-\frac{1}{\sqrt{N_{-}+1}} a_{-} \\
\frac{1}{\sqrt{\left(N_{+}+1\right)\left(N_{+}+2\right)}} a_{+}^{2} \otimes\left|0_{-}\right\rangle\left\langle 0_{-}\right|
\end{array}\right)
$$

where $\left|1_{+}, 0_{-}\right\rangle=a_{+}^{+}\left|0_{+}, 0_{-}\right\rangle$, etc. The corresponding gauge fields are

$$
\begin{aligned}
& C_{z}^{(0) a b}=\Psi_{(0)}^{a \dagger} a_{z}^{+} \Psi_{(0)}^{b}, \\
& C_{\bar{\zeta}}^{(0) a b}=\Psi_{(0)}^{a \dagger} a_{\zeta}^{+} \Psi_{(0)}^{b} .
\end{aligned}
$$

It is straightforward to check that the gauge fields $C_{z}^{(0)}, C_{\bar{\zeta}}^{(0)}$ may be obtained from vacuum by applying the solution generation technique with the following partial isometry operator

$$
S=\left(\begin{array}{cc}
0 & -\frac{1}{\sqrt{N_{-}+1}} a_{-} \\
a_{-}^{+} \frac{1}{\sqrt{N_{-}+1}} & \frac{1}{\sqrt{\left(N_{+}+1\right)\left(N_{+}+2\right)}} a_{+}^{2} \otimes\left|0_{-}\right\rangle\left\langle 0_{-}\right|
\end{array}\right)
$$


The subspace $V_{0}$ of zero vectors of this operator is two-dimensional, the basis vectors being

$$
\left|\alpha_{1}\right\rangle=\left(\begin{array}{c}
0 \\
\left|0_{+}, 0_{-}\right\rangle
\end{array}\right) \quad\left|\alpha_{2}\right\rangle=\left(\begin{array}{c}
0 \\
\left|1_{+}, 0_{-}\right\rangle
\end{array}\right)
$$

Consequently, at $\rho=0$ there is unbroken $U(2)$ gauge group on the world-volume of the two-instanton. To consider the case of non-zero $\rho$ one should note that the vectors (71) and (72) are not orthogonal at $\rho \neq 0$,

$$
\Psi^{1 \dagger} \Psi^{2}=\frac{-\sqrt{2} \rho}{\left(N_{-}+1\right)\left(N_{+}+N_{-}+2\right)\left(N_{+}+N_{-}+3\right)} a_{+}^{2} a_{-}+O\left(\rho^{2}\right) .
$$

To make them orthogonal, we replace $\Psi^{1}$ with the following linear combination

$$
\tilde{\Psi}^{1}=\left(\Psi^{1}-\Psi^{2}\left(\Psi^{2 \dagger} \Psi^{1}\right)\right) \tilde{\mathcal{N}}_{1},
$$

where $\tilde{\mathcal{N}}_{1}$ is a new normalization factor.

As follows from Eqs. (37), (39) and (41), to calculate the mass matrix of light quasi-localized modes $\psi_{\alpha}^{\beta}$ and their mixings with the continuum modes $\chi$ one does not need the explicit form of the gauge fields $C_{i}$. It is sufficient to calculate the action of these operators in the spacef $V_{0}$. It is straightforward to perform this calculation to the leading order in $\rho$, with the following result,

$$
\begin{array}{ll}
C_{+}\left|\alpha_{1}\right\rangle=\left(\begin{array}{c}
0 \\
\sqrt{2} \rho\left|1_{+}, 0_{-}\right\rangle
\end{array}\right) \quad C_{-}\left|\alpha_{1}\right\rangle=\left(\begin{array}{c}
0 \\
\sqrt{2} \rho\left|0_{+}, 1_{-}\right\rangle
\end{array}\right) \\
C_{+}\left|\alpha_{2}\right\rangle=\left(\begin{array}{c}
0 \\
\sqrt{2} \rho\left|2_{+}, 0_{-}\right\rangle
\end{array}\right) \quad C_{-}\left|\alpha_{2}\right\rangle=\left(\begin{array}{c}
0 \\
2 \rho^{2}\left|1_{+}, 1_{-}\right\rangle
\end{array}\right), \\
C_{+}^{+}\left|\alpha_{2}\right\rangle=\left(\begin{array}{c} 
\\
\sqrt{2} \rho\left|0_{+}, 0_{-}\right\rangle
\end{array}\right)
\end{array}
$$

All the rest are zero.

With these expressions at hand it is straightforward to calculate all masses and mixings of interest. Making use of Eq. (39) one obtains the following mass matrix for fields $\psi_{\alpha}^{\beta}$,

$$
\left.M_{\psi \psi}=\frac{4 \rho^{2}}{\theta}\left[\left(\psi_{1}^{1}\right)^{2}+\left(\psi_{2}^{2}\right)^{2}-\psi_{1}^{1} \psi_{2}^{2}+2 \psi_{1}^{2} \psi_{2}^{1}\right)\right]
$$

Similarly to all cases considered previously, it is straightforward to check that there is no mixing between the fields $\psi_{\alpha}^{\beta}$ and the heavy fields $\left|\xi_{\alpha}\right\rangle$ to the linear order in $\rho$. Consequently, direct mixing between the fields $\psi_{\alpha}^{\beta}$ and continuum states $\chi$, coming from the term given by Eq. (40), again gives the leading order

\footnotetext{
${ }^{4}$ Note that the operators $\Delta_{i}^{+}$entering Eqs. (37), (39) and (41) act in $V_{0}$ in the same way as the operators $C_{i}$.
} 
contribution to the widths of $\psi_{\alpha}^{\beta}$. By making use of Eqs. (78) and explicit form of the partial isometry operator (77) it is straightforward to check that the diagonal components $\psi_{1}^{1}$ and $\psi_{2}^{2}$ mix with the continuum at the order $\rho^{2}$, while mixing of the off-diagonal components $\psi_{1}^{2}=\psi_{2}^{1 *}$ occurs through $O\left(\rho^{2}\right)$ term in Eq. (78). As a result, the latter fields mix with the continuum only at the order $\rho^{3}$. Consequently, similarly to the multi-vortex case we obtain a hierarchical pattern of widths, namely

$$
\Gamma_{d} \propto \frac{\rho^{5}}{\sqrt{\theta}}
$$

for the diagonal components and

$$
\Gamma_{o} \propto \frac{\rho^{7}}{\sqrt{\theta}}
$$

for the off-diagonal components.

\section{References}

[1] C. Charmousis, R. Gregory and V. A. Rubakov, Phys. Rev. D 62 (2000) 067505 arXiv:hep-th/9912160;

R. Gregory, V. A. Rubakov and S. M. Sibiryakov, Phys. Rev. Lett. 84 (2000) 5928 arXiv:hep-th/0002072;

C. Csaki, J. Erlich and T. J. Hollowood, Phys. Rev. Lett. 84 (2000) 5932 arXiv:hep-th/0002161.

G. R. Dvali, G. Gabadadze and M. Porrati, Phys. Lett. B 484 (2000) 112 arXiv:hep-th/0002190.

[2] I. I. Kogan, S. Mouslopoulos, A. Papazoglou, G. G. Ross and J. Santiago, Nucl. Phys. B 584 (2000) 313 arXiv:hep-ph/9912552;

I. I. Kogan, S. Mouslopoulos, A. Papazoglou and G. G. Ross, Nucl. Phys. B 595 (2001) 225 arXiv:hep-th/0006030.

[3] G. R. Dvali, G. Gabadadze and M. Porrati, Phys. Lett. B 485 (2000) 208 arXiv:hep-th/0005016.

[4] G. R. Dvali, G. Gabadadze and M. A. Shifman, Phys. Lett. B 497 (2001) 271 arXiv:hep-th/0010071].

[5] S. L. Dubovsky, V. A. Rubakov and P. G. Tinyakov, Phys. Rev. D 62 (2000) 105011 arXiv:hep-th/0006046.

[6] S. L. Dubovsky, "Tunneling into extra dimension and high-energy violation of Lorentz invariance," arXiv:hep-th/0103205. 
[7] V. A. Rubakov, Uspekhi Fiz. Nauk, 171 (2001) 913, arXiv:hep-ph/0104152.

[8] N. A. Nekrasov, "Trieste lectures on solitons in noncommutative gauge theories," arXiv:hep-th/0011095.

[9] J. A. Harvey, "Komaba lectures on noncommutative solitons and D-branes," arXiv:hep-th/0102076.

[10] A. Konechny and A. Schwarz, "Introduction to M(atrix) theory and noncommutative geometry", Part I arXiv:hep-th/0012145; Part II, arXiv:hepth/0107251.

[11] A. Connes, M. R. Douglas and A. Schwarz, JHEP 9802 (1998) 003 arXiv:hep-th/9711162;

M. R. Douglas and C. M. Hull, JHEP 9802 (1998) 008 arXiv:hepth/9711165;

V. Schomerus, JHEP 9906 (1999) 030 arXiv:hep-th/9903205];

N. Seiberg and E. Witten, JHEP 9909 (1999) 032 arXiv:hep-th/9908142].

[12] L. Pilo and A. Riotto, JHEP 0103 (2001) 015 arXiv:hep-ph/0012174;

[13] J. A. Harvey, P. Kraus, F. Larsen and E. J. Martinec, JHEP 0007 (2000) 042 arXiv:hep-th/0005031.

[14] R. Gopakumar, S. Minwalla and A. Strominger, JHEP 0104 (2001) 018 arXiv:hep-th/0007226.

[15] M. R. Douglas, "Branes within branes," arXiv:hep-th/9512077.

[16] A. P. Polychronakos, Phys. Lett. B 495 (2000) 407 arXiv:hep-th/0007043.

[17] D. P. Jatkar, G. Mandal and S. R. Wadia, JHEP 0009 (2000) 018 arXiv:hepth/0007078.

[18] D. Bak, Phys. Lett. B 495 (2000) 251 arXiv:hep-th/0008204.

[19] D. Bak, K. Lee and J. H. Park, Phys. Rev. D 63 (2001) 125010 arXiv:hepth/0011099.

[20] N. Nekrasov and A. Schwarz, Commun. Math. Phys. 198 (1998) 689 arXiv:hep-th/9802068.

[21] M. Aganagic, R. Gopakumar, S. Minwalla and A. Strominger, JHEP 0104 (2001) 001 arXiv:hep-th/0009142.

[22] K. Furuuchi, "Topological charge of U(1) instantons on noncommutative $\mathrm{R}^{* *} 4$," arXiv:hep-th/0010006. 
[23] J. A. Harvey, P. Kraus and F. Larsen, JHEP 0012 (2000) 024 arXiv:hepth/0010060.

[24] K. Furuuchi, JHEP 0103 (2001) 033 arXiv:hep-th/0010119.

[25] S. L. Dubovsky, V. A. Rubakov and P. G. Tinyakov, JHEP 0008 (2000) 041 arXiv:hep-ph/0007179.

[26] M. Hamanaka, ADHM/Nahm construction of localized solitons in noncommutative gauge theories, hep-th/0109070.

[27] M. F. Atiyah, N. J. Hitchin, V. G. Drinfeld and Y. I. Manin, Phys. Lett. A 65, 185 (1978). 